\title{
Pulmonary Artery Angiosarcoma
}

National Cancer Institute

\section{Source}

National Cancer Institute. Pulmonary Artery Angiosarcoma. NCI Thesaurus. Code C6746.

A malignant vascular neoplasm arising from the pulmonary artery. 